UCRL-JC-121960

PREPRINT

\title{
Molecular Dynamics Simulation of Shocks in Porous TATB Crystals
}

L. E. Fried

C. Tarver

$$
\begin{gathered}
\text { Finut } \\
\text { APR } 051995 \\
\text { OSTI }
\end{gathered}
$$

This paper was prepared for submittal to the 1995 APS Topical Conference

Seattle, Washington

August 13-18, 1995

\section{August 1995}

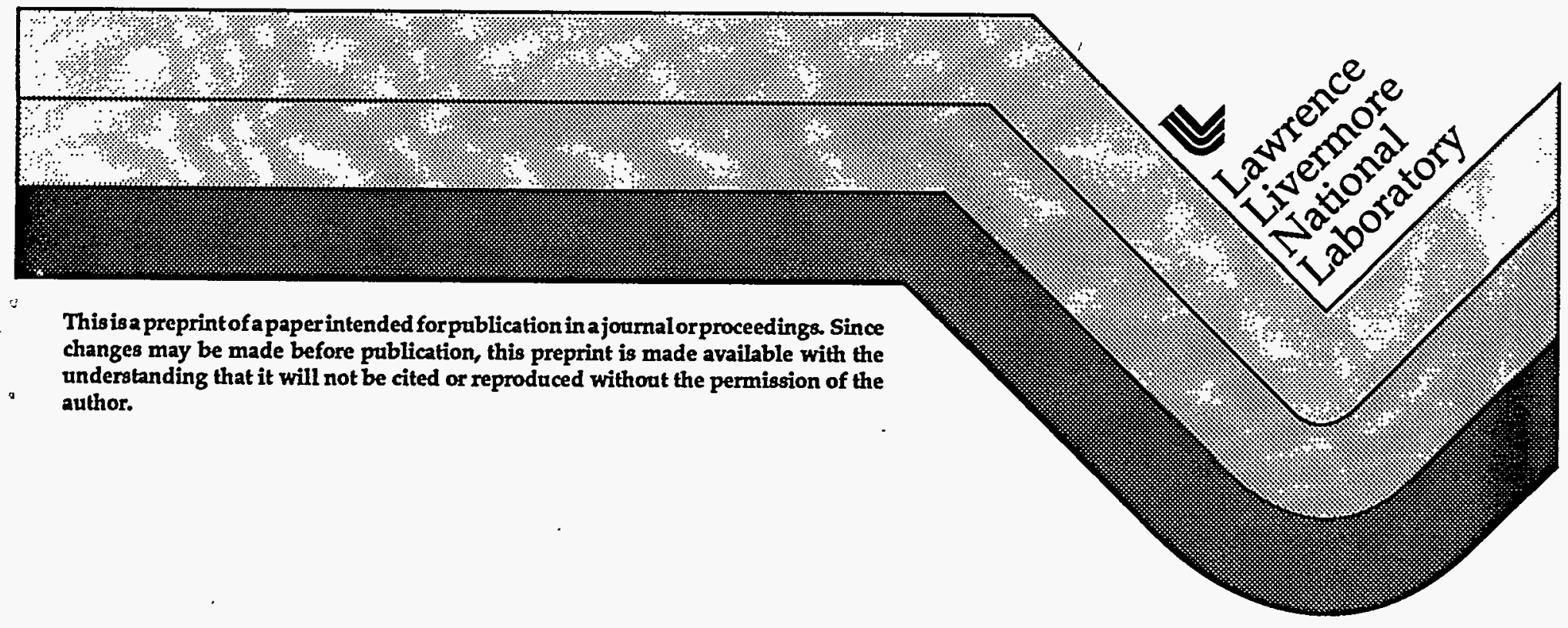




\section{DISCLAIMER}

This document was prepared as an account of work sponsored by an agency of the United States Government. Neither the United States Government nor the University of California nor any of their employees, makes any warranty, express or implied, or assumes any legal liability or responsibility for the accuracy, completeness, or usefulness of any information, apparatus, product, or process disclosed, or represents that its use would not infringe privately owned rights. Reference herein to any specific commercial product, process, or service by trade name, trademark, manufacturer, or otherwise, does not necessarily constitute or imply its endorsement, recommendation, or favoring by the United States Government or the University of California. The views and opinions of authors expressed herein do not necessarily state or reflect those of the United States Government or the University of California, and shall not be used for advertising or product endorsement purposes. 


\title{
MOLECULAR DYNAMICS SIMULATION OF SHOCKS IN POROUS TATB CRYSTALS
}

\author{
Laurence E. Fried and Craig Tarver. \\ L-282, Energetic Materials Center, Lawrence Livermore National Laboratory, Livermore, CA 94550
}

\begin{abstract}
We report molecular dynamics results on the shock structure of two dimensional crystals of triaminotrinitrobenzene (TATB). We find that the shock front broadens to approximately $30 \mathrm{~nm}$ in materials with a 20 percent random void distribution. As expected from bulk experiments, the shock velocity decreases with increasing porosity and the temperature behind the shock front increases with increasing porosity. Shock equilibration times increase from 1 ps to greater than 10 ps.
\end{abstract}

\section{INTRODUCTION}

Over the past decade molecular dynamics simulations have been applied to shocks in a range of materials. In one of the earliest calculations in the field, Tsai ${ }^{1}$ studied shocks in Lennard-Jones crystals. Hoover ${ }^{2}$ and coworkers performed simulations of shocks in a simple Lennard-Jones fluid. Karo performed a study of detonation for a model system where energy was directly released upon reaction. White, Brenner, Robertson, and coworkers ${ }^{4}$ at the Naval Research Laboratory performed an interesting series of simulations of two and three dimensional reacting diatomics. This work employed an empirical bond-order potential to describe reactions. Self-sustaining detonations were found to occur in the simulations. More recently members of the Naval Research Laboratory group have simulated shocks in ozone crystals ${ }^{5}$.

Energetic materials used in military applications are typically large molecules containing several nitro groups. Good examples are tri-nitrotoluene (TNT) and tri-nitrotriaminobenzene (TATB). Many common energetic materials have over twenty atoms. The shock dynamics of large floppy molecules is expected to be different from the shock dynamics of diatomics and triatomics.

One simple difference is found in the large number of vibrational modes. TATB, for example, has 66 vibrations. At the shock front, however, all energy is channeled into translations. This leads to a huge translational temperature overshoot at the shock front. For detonation shocks this overshoot can be as large as $20,000 \mathrm{~K}^{6}$. A second difference between small and large molecules is that most large molecules have many low frequency vibrational modes. TATB has ring modes as low as $50 \mathrm{~cm}^{-1}$. Low frequency molecular vibrations can accept energy from translational modes at the shock front without an intervening anharmonic process. This leads to very fast transfer of energy from the shock front to the low frequency molecular modes.

The simulation of large molecules such as TATB poses several computational challenges. To begin with, the intramolecular potential energy surface becomes more complicated. In addition to the two-body forces that dominate most simple fluids, molecular systems have important three and four body interactions. The most important of these interactions are bond angle bending and torsional forces. While bond stretches typically have freqencies over $1000 \mathrm{~cm}^{-1}$, bend motions have frequencies of several hundred $\mathrm{cm}^{-1}$. Torsional frequencies are often under $100 \mathrm{~cm}^{-1}$. $^{\circ}$ Therefore it is the magnitude of the three and four body intramolecular interactions that make large molecules floppy.

A second difficulty with large molecules is that they can undergo a variety of chemical reactions. Each product molecule can then undergo its own complicated set of chemical reactions. The long chain of possible reactions and the substantial difficulty of generating a reliable potential surface for a single reaction puts the simulation of detonating waves of most common energetic 
materials outside of the range of current computational facilities and numerical methods.

In the present paper we will simulate shocks in non-reacting TATB. TATB has a shock initiation threshold of $3 \mathrm{~km} / \mathrm{s}$ or above, depending on its preparation and porosity. We will study the propagation of shocks with particle velocities of 1 $\mathrm{km} / \mathrm{s}$ or less for a time period of $10 \mathrm{ps}$. Given the shock initiation threshold of TATB, we expect that there will not be significant chemical reaction during the timespan of the simulations.

\section{SIMULATION METHOD}

\section{Force model}

All the atoms of TATB were included in the simulation. The intramolecular forces included harmonic bond stretching, a $\cos (\theta)^{2}$ bend interaction and $\cos (\varphi)$ torsional interaction. The functional forms and parameters were taken from the DREIDNG $^{7}$ molecular mechanics potential energy surface.

Lennard-Jones interactions were used for the intermolecular forces. Since TATB is a nonpolar molecule, Coulombic interactions were not included in the simulation. The intermolecular interactions were cut off at $20 \mathrm{~nm}$. This is roughly 5 times the Lennard-Jones $\sigma$. A switching function was used to smoothly tum the interaction off at large distances, thus providing improved energy conservation.

\section{Propagation method}

Trajectories were propagated with the velocity version of the Verlet algorithm. A timestep of 0.5 fs was used in the simulations. Velocities were scaled once every 20 steps to ensure energy conservation. The simulation cell was two dimensional, with periodic boundary conditions enforced transverse to the shock front. Free boundary conditions were employed in the direction of the shock front. Shocks were initiated by a flyer plate consisting of two rows of highly massive $\left(\mathrm{MW}=10^{4}\right)$ small $(\sigma=0.1 \mathrm{~nm})$ LennardJones particles.

\section{Initial Conditions}

We simulated two dimensional TATB crystals. The crystal structure of TATB consists of overlapping planes of molecules. Each plane is nearly (within a few degrees) a two dimensional hexagonal plane. Thus, TATB naturally maps into a two dimensional model. In the simulation the TATB molecules were arranged in a hexagonal lattice. The lattice spacing was varied so as to minimize the energy. This led to a lattice spacing of $0.95 \mathrm{~nm}$. In order to clearly separate the effects of thermal and lattice disorder, the TATB material was given an initial temperature of $0 \mathrm{~K}$. Further studies will investigate shocks in thermal TATB.

Porous TATB samples were achieved by randomly removing molecules with a given probability. This is a simplified model of a unimolecular decomposition process, where the TATB has decomposed into products small enough to diffuse out of the crystal lattice.

\section{RESULTS}

We investigated the shock structure of perfect crystals of TATB first. We found it sufficient to use a TATB crystal with 10 lattice spacings in the transverse $(y)$ direction and 40 lattice spacings in the shock ( $x$ ) direction. The shock velocity achieves a steady state with 1 ps. This is shown in Fig. 1, where the shock position is plotted as a function of time. The shock position was tracked by monitoring the temperature discontinuity as a function of time.

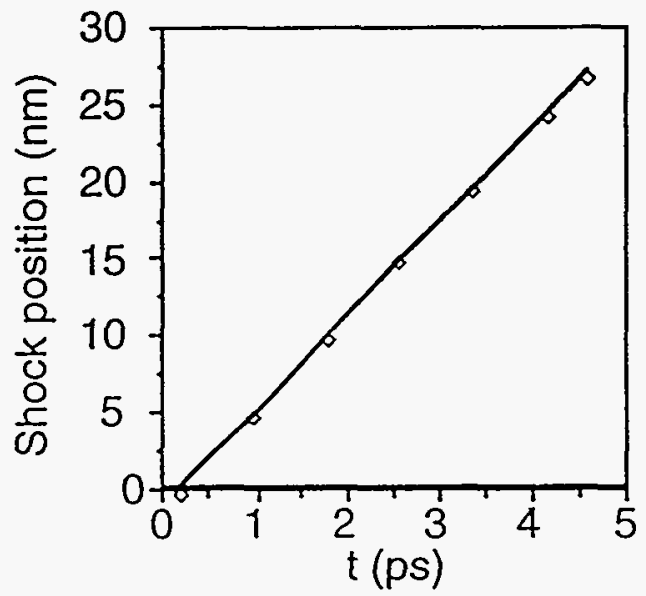

FIGURE 1. The shock position vs. time for purc TATB. 
We next proceeded to find the shock.Hugoniot of a perfect TATB crystal in two dimensions. Since TATB is a highly asymmetric crystal, one would expect the 2-D shock Hugoniot to depend strongly on the plane chosen to define the 2-D system. In our case, we have chosen the plane to be along a direction of low compressibility. Hence sound speeds and shock propagation are expected . to be higher than an orientationally averaged experimental value. In Fig. 2 we show the shock Hugoniot calculated for perfect 2-D TATB crystals.

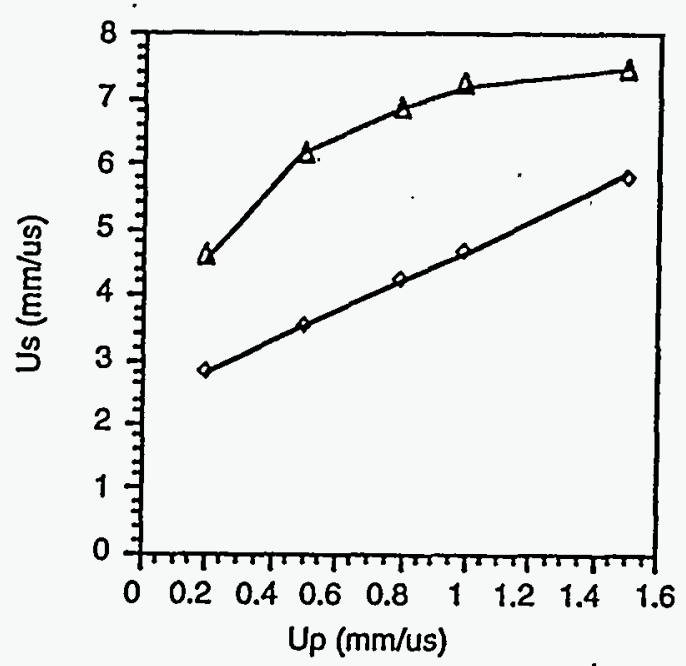

FIGURE 2: The calculated 2-D (upper) and experimental (lower) shock Hugoniot of TATB.

We next consider the propagation of shocks in porous TATB. In these simulations molecules were randomly removed from the 2-D lattice with a specified probability. The particle velocity in these simulations was $\mathrm{i} \mathrm{mm} / \mu \mathrm{s}$. Figure 3 shows a snapshot of a $10 \mathrm{ps}$ simulation. A simulation cell of $75(x)$ by $19(y) \mathrm{nm}$ was used. The shock front is spread out over roughly $10 \mathrm{~nm}$. The collapse of voids leads to the production of hot spots and a highly non-uniform temperature distribution behind the shock front. Similar effects are seen in $40 \%$ porous TATB. A 75 by $75 \mathrm{~nm}$ simulation cell was used in this case to obtain better averaging over the many pore sizes. A snapshot is shown in Fig. 4 at 10 ps. The shock speed is much lower in the highly porous material. The shock front is very broad. Equilibration of the system into a well-defined shock front and steady state zone behind the front has not yet occurred.
It is interesting to compare the effect that porosity has on shock temperatures. The increased temperatures caused by void compression (referred to as hot spots in the explosives literature) lead to high average temperatures in the porous material. In Fig. 5 we show shock temperatures for $20 \%$ porous and $40 \%$ porous TATB.The increase in temperature with porosity is a well known effect. Porosity increases the average temperature behind the shock front by nearly a factor of two.
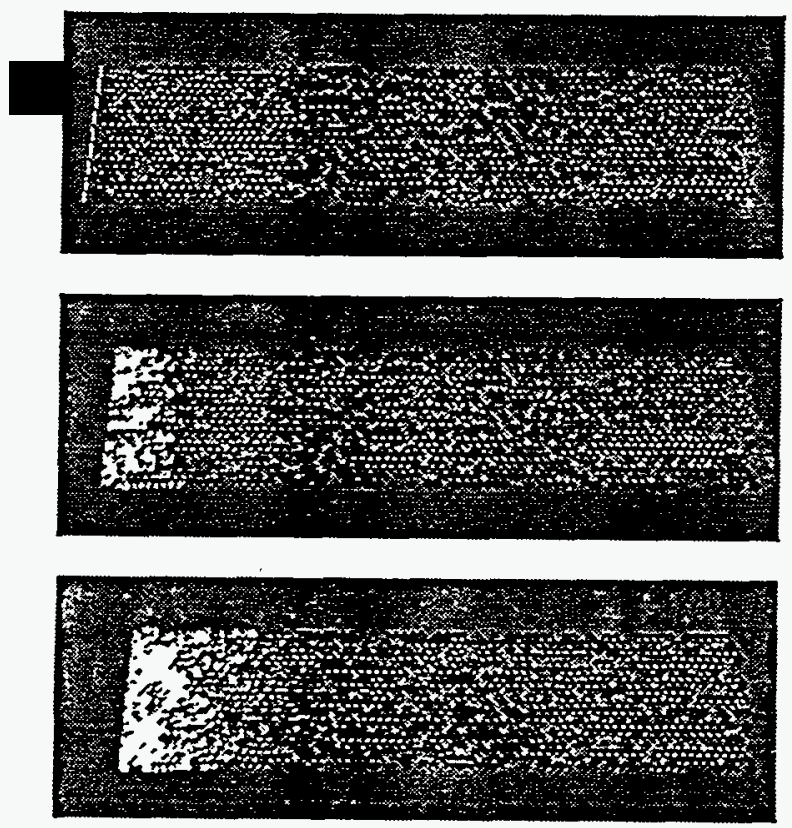

FIGURE 3. A simulation of $20 \%$ porous TATB is shown at time of 0 (top) 2.5 (middle) and 5 (bottom) ps. The molecules are shaded according to their kinetic energy. Pure white corresponds to $800 \mathrm{~K}$.

The break in slope in the $20 \%$ porosity temperature distribution shows that equilibration to a steady shock front has begun within the $10 \mathrm{ps}$ simulation time. There is no such break in the $40 \%$ porosity distribution, indicating that equilibration times are much longer than 10ps.

There is a broad distribution of particle velocity accompanying the temperature distribution in the porous materials. In Fig. 6 we show the particle velocity as a function of position for 20 and $40 \%$ porous

TATB. 


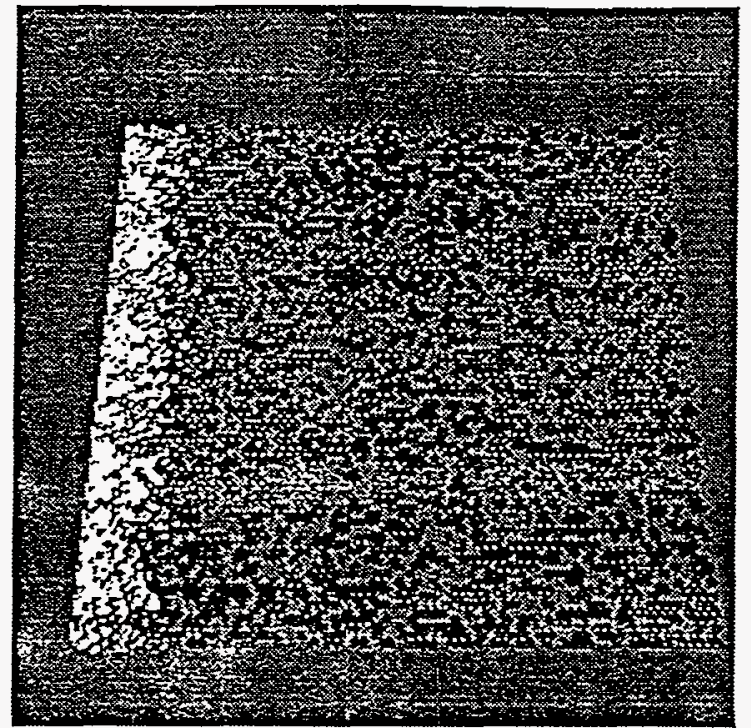

FIGURE 4: A simulation of $40 \%$ porous TATB is shown at a time of 10 ps. The molecules are shaded according to their

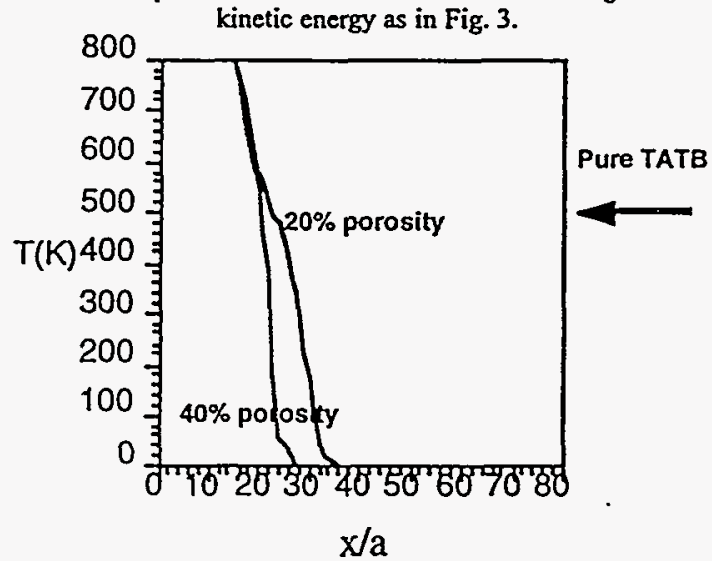

FIGURE 5. The temperature profile across the shock front is shown for simulations of $20 \%$ and $40 \%$ porous TATB. The calculated shock temperature of pure TATB is shown for reference. Distance is shown in units of one lattice spacing.

\section{DISCUSSION}

In this paper we have described how shock properties of two dimensional TATB crystals change as a function of random porosity. The most dramatic effects seen are a factor of 4 decrease in the shock velocity and a factor of 10 or more increase in the shock equilibration time. Temperatures behind the shock front increased by roughly a factor of two. Furture work on this project will involve better TATB potential models, three dimensional shocks, and early time chemical reactions

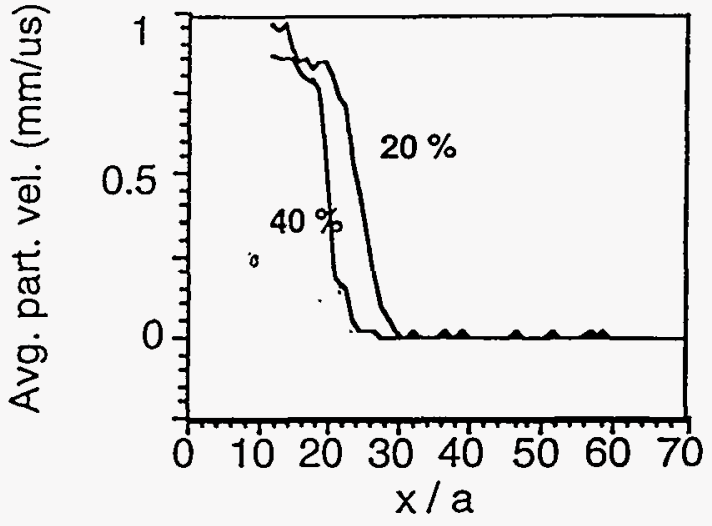

FIGURE 6: The average particle velocity across the shock front is shown as a function of distance for $20 \%$ porous and $40 \%$ porous TATB.

Ackowledgements

Research described in this article was performed under the auspices of the US Department of Energy by Lawrence Livermore National Laboratory under contract No. W-7405-ENG-48.

\section{REFERENCES}

1. Tsai, D.H. and MacDonald, R.A., Phys. Rev. B, 14, 47144723(1976).

2. Hoover, W.G., Phys. Rev, Lett, 42, 1531-1534(1979).

3. Karo, A.M., Handy, J.R., and Walker, F.E., Acta Astronautica 5, 1041-1062(1978).

4. Brenner, D.W., Robertson, D.H., Elert, M.H., and White, C.T., Phys. Rev. Lett, 70, 2174(1993).

5. Barrett, J.J.C., Whitc, C.T., Brenner, D.W., and Robertson, D.H., "Detonics of Solid $\mathrm{O}_{3}$ : Effects of Void Collapse", paper presented at this symposium

6. Tarver, C.M. Combustion and Flame, 46, 157-176 (1982).

7. Mayo, S.L., Olafson, B.D., Goddard, W.A. III, J. Phys. Chem. 94, 8897 (1990).

8. Honodel, C.A., Humphrey, J.R., Weingart, R.C., Loe, R.S., and Kramer, P., Seveath Symposium (International) on Detonation, Naval Surface Weapons Center NSWCMP.82-334, Annapolis, MD, p. 425-434.

9. Zeldovich, Y.B. and Raizer, Y.P., Physics of Shock Waves and High-Temperature Hydrodynamic Phenomena, Academic Press, 1967. Chapter XI. 
$\cdots-\cdots+\ldots$ 


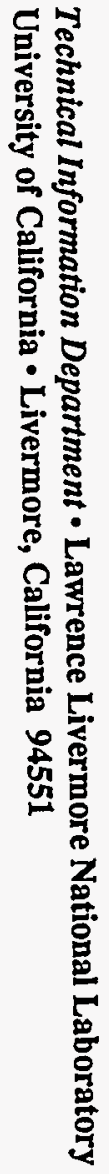

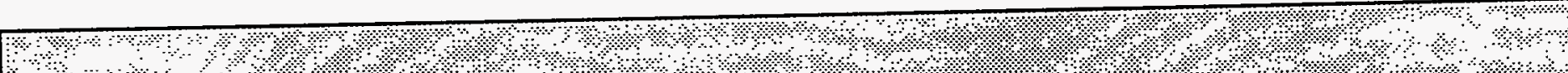

